\title{
A SUB-RIEMANNIAN MAXIMUM PRINCIPLE AND ITS APPLICATION TO THE $p$-LAPLACIAN IN CARNOT GROUPS
}

\author{
Thomas Bieske \\ University of South Florida, Department of Mathematics and Statistics \\ Tampa, FL 33620, U.S.A.; tbieske@math.usf.edu
}

\begin{abstract}
We prove a sub-Riemannian maximum principle for semicontinuous functions. We apply this principle to Carnot groups to provide a "sub-Riemannian" proof of the uniqueness of viscosity infinite harmonic functions. This is an alternate method of proof from the one found in [15]. We also establish the equivalence of weak solutions and viscosity solutions to the $p$-Laplace equation. This result extends the author's previous work in the Heisenberg group [3, 4].
\end{abstract}

\section{Calculus on Carnot groups}

We begin by denoting an arbitrary Carnot group in $\mathbf{R}^{N}$ by $G$ and its corresponding Lie Algebra by $g$. Recall that $g$ is nilpotent and stratified, resulting in the decomposition

$$
g=V_{1} \oplus V_{2} \oplus \cdots \oplus V_{l}
$$

for appropriate vector spaces that satisfy the Lie bracket relation $\left[V_{1}, V_{j}\right]=V_{1+j}$. The Lie Algebra $g$ is associated with the group $G$ via the exponential map exp: $g \rightarrow G$. Since this map is a diffeomorphism, we can choose a basis for $g$ so that it is the identity map. Denote this basis by

$$
X_{1}, X_{2}, \ldots, X_{n_{1}}, Y_{1}, Y_{2}, \ldots, Y_{n_{2}}, Z_{1}, Z_{2}, \ldots, Z_{n_{3}}
$$

so that

$$
\begin{aligned}
V_{1} & =\operatorname{span}\left\{X_{1}, X_{2}, \ldots, X_{n_{1}}\right\}, \\
V_{2} & =\operatorname{span}\left\{Y_{1}, Y_{2}, \ldots, Y_{n_{2}}\right\}, \\
V_{3} \oplus V_{4} \oplus \cdots \oplus V_{l} & =\operatorname{span}\left\{Z_{1}, Z_{2}, \ldots, Z_{n_{3}}\right\} .
\end{aligned}
$$

We endow $g$ with an inner product $\langle\cdot, \cdot\rangle$ and related norm $\|\cdot\|$ so that this basis is orthonormal. Clearly, the Riemannian dimension of $g$ (and so $G$ ) is $N=n_{1}+n_{2}+n_{3}$. However, we will also consider the homogeneous dimension of $G$, denoted $\mathcal{Q}$, which is given by

$$
\mathcal{Q}=\sum_{i=1}^{l} i \cdot \operatorname{dim} V_{i}
$$

doi:10.5186/aasfm.2012.3706

2010 Mathematics Subject Classification: Primary 53C17, 35D40, 31C45, 35H20; Secondary 31B05, 22E25.

Key words: Sub-Riemannian geometry, non-linear potential theory, viscosity solutions, $p$ Laplacian. 
Before proceeding with the calculus, we recall the group and metric space properties. Since the exponential map is the identity, the group law is the CampbellHausdorff formula (see, for example, [5]). For our purposes, this formula is given by

$$
p \cdot q=p+q+\frac{1}{2}[p, q]+R(p, q)
$$

where $R(p, q)$ are terms of order 3 or higher. The identity element of $G$ will be denoted by 0 and called the origin. There is also a natural metric on $G$, which is the Carnot-Carathéodory distance, defined for the points $p$ and $q$ as follows:

$$
d_{C}(p, q)=\inf _{\Gamma} \int_{0}^{1}\left\|\gamma^{\prime}(t)\right\| d t
$$

where the set $\Gamma$ is the set of all curves $\gamma$ such that $\gamma(0)=p, \gamma(1)=q$ and $\gamma^{\prime}(t) \in V_{1}$. By Chow's theorem (see, for example, [2]) any two points can be connected by such a curve, which means $d_{C}(p, q)$ is an honest metric. Define a Carnot-Carathéodory ball of radius $r$ centered at a point $p_{0}$ by

$$
B\left(p_{0}, r\right)=\left\{p \in G: d_{C}\left(p, p_{0}\right)<r\right\} .
$$

In addition to the Carnot-Carathéodory metric, there is a smooth (off the origin) gauge. This gauge is defined for a point $p=\left(\zeta_{1}, \zeta_{2}, \ldots, \zeta_{l}\right)$ with $\zeta_{i} \in V_{i}$ by

$$
\mathcal{N}(p)=\left(\sum_{i=1}^{l}\left\|\zeta_{i}\right\|^{\frac{2 l !}{i}}\right)^{\frac{1}{2 l !}}
$$

and it induces a distance $d_{\mathcal{N}}$ given by

$$
d_{\mathcal{N}}(p, q)=\mathcal{N}\left(p^{-1} \cdot q\right)
$$

This distance is bi-Lipschitz equivalent to the Carnot-Carathéodory metric [2]. We define a gauge ball of radius $r$ centered at a point $p_{0}$ by

$$
B_{\mathcal{N}}\left(p_{0}, r\right)=\left\{p \in G: d_{\mathcal{N}}\left(p, p_{0}\right)<r\right\} .
$$

In this environment, a smooth function $u: G \rightarrow \mathbf{R}$ has the horizontal derivative given by

$$
\nabla_{0} u=\left(X_{1} u, X_{2} u, \ldots, X_{n_{1}} u\right)
$$

and the symmetrized horizontal second derivative matrix, denoted by $\left(D^{2} u\right)^{\star}$, with entries

$$
\left(\left(D^{2} u\right)^{\star}\right)_{i j}=\frac{1}{2}\left(X_{i} X_{j} u+X_{j} X_{i} u\right)
$$

for $i, j=1,2, \ldots, n_{1}$. We also consider the semi-horizontal derivative given by

$$
\nabla_{1} u=\left(X_{1} u, X_{2} u, \ldots, X_{n_{1}} u, Y_{1} u, Y_{2} u, \ldots, Y_{n_{2}} u\right) \text {. }
$$

We recall that for any open set $\mathcal{O} \subset G$, the function $f$ is in the horizontal Sobolev space $W^{1, P}(\mathcal{O})$ if $f$ and $X_{i} f$ are in $L^{P}(\mathcal{O})$ for $i=1,2, \ldots, n_{1}$. Replacing $L^{P}(\mathcal{O})$ by $L_{l o c}^{P}(\mathcal{O})$, the space $W_{l o c}^{1, P}(\mathcal{O})$ is defined similarly. The space $W_{0}^{1, P}(\mathcal{O})$ is the closure in $W^{1, P}(\mathcal{O})$ of smooth functions with compact support. In addition, we recall a function $u: G \rightarrow \mathbf{R}$ is $\mathcal{C}_{\text {sub }}^{2}$ if $\nabla_{1} u$ and $X_{i} X_{j} u$ are continuous for all $i, j=1,2, \ldots, n_{1}$. Note that $\mathcal{C}_{\text {sub }}^{2}$ is different from the regular (Euclidean) $C^{2}$. 
Using the above derivatives, we define the horizontal $p$-Laplacian of a smooth function $f$ for $1<p<\infty$ by

$$
\begin{aligned}
\Delta_{p} f & =\operatorname{div}\left(\left\|\nabla_{0} f\right\|^{p-2} \nabla_{0} f\right)=\sum_{i=1}^{n_{1}} X_{i}\left(\left\|\nabla_{0} f\right\|^{p-2} \nabla_{0} f\right) \\
& =\left\|\nabla_{0} f\right\|^{p-2} \operatorname{tr}\left(\left(D^{2} f\right)^{\star}\right)+(p-2)\left\|\nabla_{0} f\right\|^{p-4}\left\langle\left(D^{2} f\right)^{\star} \nabla_{0} f, \nabla_{0} f\right\rangle .
\end{aligned}
$$

Formally taking the limit as $p$ goes to infinity results in the infinite Laplacian which is defined by

$$
\Delta_{\infty} f=\sum_{i, j=1}^{n_{1}} X_{i} f X_{j} f X_{i} X_{j} f=\left\langle\left(D^{2} f\right)^{\star} \nabla_{0} f, \nabla_{0} f\right\rangle .
$$

\section{Carnot jets and viscosity solutions}

We begin by recalling the following Taylor theorem [9]:

Theorem 2.1. For a smooth function $u: G \rightarrow \mathbf{R}$, we have the following Taylor formula at the point $p_{0}$ :

$$
u(p)=u\left(p_{0}\right)+\left\langle\nabla_{1} u\left(p_{0}\right), \widehat{p_{0}^{-1} p}\right\rangle+\frac{1}{2}\left\langle\left(D^{2} u\left(p_{0}\right)\right)^{\star} \overline{p_{0}^{-1} p}, \overline{p_{0}^{-1} p}\right\rangle+o\left(\left(d\left(p_{0}, p\right)\right)^{2}\right)
$$

where $\overline{p_{0}^{-1} p}$ is the projection of $p_{0}^{-1} p$ onto $V_{1}$ and $\widehat{p_{0}^{-1}} p$ is the projection of $p_{0}^{-1} p$ onto $V_{1} \oplus V_{2}$. (Recall that exp is the identity map.)

This theorem motivates the following definition for the semi-jets.

Definition 1. Let $u$ be an upper semicontinuous function $u: G \rightarrow \mathbf{R}$ and let $S^{n_{1}}$ be the set of all $n_{1} \times n_{1}$ symmetric matrices. For $\eta \in V_{1} \oplus V_{2}$ and $X \in S^{n_{1}}$, consider the following inequality:

$$
u(p) \leq u\left(p_{0}\right)+\left\langle\eta, \widehat{p_{0}^{-1}} p\right\rangle+\frac{1}{2}\left\langle X \overline{p_{0}^{-1} p}, \overline{p_{0}^{-1} p}\right\rangle+o\left(\left(d\left(p_{0}, p\right)\right)^{2}\right) \text { as } p \rightarrow p_{0} .
$$

The second-order superjet of $u$ at $p_{0}$, denoted $J^{2,+} u\left(p_{0}\right)$, is given by

$$
J^{2,+} u\left(p_{0}\right)=\left\{(\eta, X) \subset\left(V_{1} \oplus V_{2}\right) \times S^{n_{1}} \text { : equation (2.1) holds }\right\} .
$$

The second-order subjet of the lower semicontinuous function $v$ at $p_{0}$, denoted by $J^{2,-} v\left(p_{0}\right)$, is defined by

$$
J^{2,-} v\left(p_{0}\right)=-J^{2,+}(-v)\left(p_{0}\right) .
$$

We define the set-theoretic closure of $J^{2,+} u\left(p_{0}\right)$, denoted $\bar{J}^{2,+} u\left(p_{0}\right)$ by $(\eta, X) \in$ $\bar{J}^{2,+} u\left(p_{0}\right)$ if there exists a sequence $\left\{\left(p_{m}, u\left(p_{m}\right), \eta_{m}, X_{m}\right)\right\} \in G \times \mathbf{R} \times g \times S^{n_{1}}$ so that as $m \rightarrow \infty$, we have $\left\{\left(p_{m}, u\left(p_{m}\right), \eta_{m}, X_{m}\right)\right\} \rightarrow\left(p_{0}, u\left(p_{0}\right), \eta, X\right)$ with $\left(\eta_{m}, X_{m}\right) \in$ $J^{2,+} u\left(p_{m}\right)$.

Given the upper semicontinuous function $u$, we may define the set of test functions that touch $u$ from above at $p_{0}$, denoted $\mathcal{T} \mathcal{A}\left(u, p_{0}\right)$ and given a lower semicontinuous function $v$, we may define the set of test functions that touch $v$ from below at $p_{0}$, denoted $\mathcal{T} \mathcal{B}\left(v, p_{0}\right)$. Namely,

$\mathcal{T} \mathcal{A}\left(u, p_{0}\right)=\left\{\phi: G \rightarrow \mathbf{R}: \phi \in \mathcal{C}_{\text {sub }}^{2}\left(p_{0}\right), \phi\left(p_{0}\right)=u\left(p_{0}\right)\right.$ and $\phi(p)>u(p)$ for $p$ near $\left.p_{0}\right\}$, $\mathcal{T} \mathcal{B}\left(v, p_{0}\right)=\left\{\phi: G \rightarrow \mathbf{R}: \phi \in \mathcal{C}_{\text {sub }}^{2}\left(p_{0}\right), \phi\left(p_{0}\right)=v\left(p_{0}\right)\right.$ and $\phi(p)<v(p)$ for $p$ near $\left.p_{0}\right\}$. 
We define the sets $K^{2,+} u\left(p_{0}\right)$ and $K^{2,-} v\left(p_{0}\right)$ by

$$
\begin{aligned}
& K^{2,+} u\left(p_{0}\right)=\left\{\left(\nabla_{1} \phi\left(p_{0}\right),\left(D^{2} \phi\left(p_{0}\right)\right)^{\star}\right): \phi \in \mathcal{T} \mathcal{A}\left(u, p_{0}\right)\right\}, \\
& K^{2,-} v\left(p_{0}\right)=\left\{\left(\nabla_{1} \phi\left(p_{0}\right),\left(D^{2} \phi\left(p_{0}\right)\right)^{\star}\right): \phi \in \mathcal{T} \mathcal{B}\left(v, p_{0}\right)\right\} .
\end{aligned}
$$

As the following lemma shows, the two sets $J^{2,+} u\left(p_{0}\right)$ and $K^{2,+} u\left(p_{0}\right)$ are indeed the same.

Lemma 2.2. Given an upper semicontinuous function $u$ and lower semicontinuous function $v$, we have

$$
J^{2,+} u\left(p_{0}\right)=K^{2,+} u\left(p_{0}\right) \text { and } J^{2,-} v\left(p_{0}\right)=K^{2,-} v\left(p_{0}\right) .
$$

Proof. The containments $K^{2,+} u\left(p_{0}\right) \subset J^{2,+} u\left(p_{0}\right)$ and $K^{2,-} v\left(p_{0}\right) \subset J^{2,-} v\left(p_{0}\right)$ are clear. The opposite containments follow the proof of the Euclidean version of this lemma, as found in [7]. The only adjustment is to replace the Euclidean distance with the smooth Carnot norm $\mathcal{N}$. See [3] for details in the case of the Heisenberg group.

We use these jets to define viscosity solutions to the class of equations given by:

$$
F\left(p, u(p), \nabla_{1} u(p),\left(D^{2} u(p)\right)^{\star}\right)=0
$$

where the function

$$
F: G \times \mathbf{R} \times g \times S^{n_{1}} \rightarrow \mathbf{R}
$$

satisfies

$$
F(p, r, \eta, X) \leq F(p, s, \eta, Y)
$$

when $r \leq s$ and $Y \leq X$. In the language of viscosity solutions we say that $F$ is proper [8]. Examples of such equations include the $p$-Laplace equation, defined for $1<p<\infty$, by

$$
-\left(\left\|\nabla_{0} u\right\|^{p-2} \operatorname{tr}\left(\left(D^{2} u\right)^{\star}\right)+(p-2)\left\|\nabla_{0} u\right\|^{p-4}\left\langle\left(D^{2} u\right)^{\star} \nabla_{0} u, \nabla_{0} u\right\rangle\right)=0
$$

and the infinite Laplace equation

$$
-\left\langle\left(D^{2} u\right)^{\star} \nabla_{0} u, \nabla_{0} u\right\rangle=0 .
$$

\section{Sub-Riemannian maximum principle}

We recall that the set $V_{1}=\operatorname{span}\left\{X_{1}, X_{2}, \ldots, X_{n_{1}}\right\}$ and notationally, we will always denote $n_{1}$ by $n$. The vectors $X_{i}$ at the point $p \in G$ can be written as

$$
X_{i}(p)=\sum_{j=1}^{N} a_{i j}(p) \frac{\partial}{\partial x_{j}}
$$

forming the $n \times N$ matrix A with smooth entries $\mathbf{A}_{i j}=a_{i j}(p)$. By linear independence of the $X_{i}$, A has rank $n$. Similarly,

$$
Y_{i}(p)=\sum_{j=1}^{N} b_{i j}(p) \frac{\partial}{\partial x_{j}}
$$

forming the $n_{2} \times N$ matrix $\mathbf{B}$ with smooth entries $\mathbf{B}_{i j}=b_{i j}(p)$. The matrix $\mathbf{B}$ has rank $n_{2}$. 
We now state Lemma 3 from [1], which was discussed only in the case $n=N$. This lemma is actually valid in any sub-Riemannian environment. The proof for the case $n<N$ is identical and omitted.

Lemma 3.1. [1, Lemma 3] Let $u$ be a smooth function with $\nabla_{\text {eucl }} u$ its Euclidean gradient and let $D_{\text {eucl }}^{2} u$ be the Euclidean second-order derivative matrix of $u$. Let $\mathbf{A}^{T}$ denote the transpose of the matrix $\mathbf{A}$ and $\left(\mathbf{A}^{T} \cdot t\right)_{k}$ denote the $k$-th component of the vector $\mathbf{A}^{T} \cdot t$. Then, we have

$$
\nabla_{1} u=\mathbf{A} \cdot \nabla_{\text {eucl }} u \oplus \mathbf{B} \cdot \nabla_{\text {eucl }} u
$$

and for all $t \in \mathbf{R}^{n}$

$$
\begin{aligned}
\left\langle\left(D^{2} u\right)^{\star} \cdot t, t\right\rangle & =\left\langle\mathbf{A} \cdot D_{\text {eucl }}^{2} u \cdot \mathbf{A}^{T} \cdot t, t\right\rangle+\sum_{k=1}^{N}\left\langle\mathbf{A}^{T} \cdot t, \nabla_{\text {eucl }}\left(\mathbf{A}^{T} \cdot t\right)_{k}\right\rangle \frac{\partial u}{\partial x_{k}} \\
& =\left\langle\left(\mathbf{A} \cdot D_{\text {eucl }}^{2} u \cdot \mathbf{A}^{T}+\mathbf{M}\left(\nabla_{\text {eucl }}(p), p\right)\right) \cdot t, t\right\rangle
\end{aligned}
$$

where the entries of the (symmetric) $n \times n$ matrix $\mathbf{M}\left(\nabla_{\text {eucl }}(p), p\right)$ are given by

$$
\mathbf{M}\left(\nabla_{\text {eucl }}(p), p\right)_{i j}= \begin{cases}\frac{1}{2} \sum_{k=1}^{N} \sum_{l=1}^{N}\left(a_{i l}(p) \frac{\partial a_{j k}}{\partial x_{l}}(p)+a_{j l}(p) \frac{\partial a_{i k}}{\partial x_{l}}(p)\right) \frac{\partial u}{\partial x_{k}}, & i \neq j, \\ \sum_{k=1}^{N} \sum_{l=1}^{N} a_{i l}(p) \frac{\partial a_{i k}}{\partial x_{l}}(p) \frac{\partial u}{\partial x_{k}}, & i=j .\end{cases}
$$

This result coupled with Lemma 2.2 produces the following corollary:

Corollary 3.2. Let $(\eta, X) \in \bar{J}_{\text {eucl }}^{2,+} u(p)$. (Recall that $(\eta, X) \in \mathbf{R}^{N} \times S^{N}$.) Then

$$
\left(\mathbf{A} \cdot \eta \oplus \mathbf{B} \cdot \eta, \mathbf{A} X \mathbf{A}^{T}+\mathbf{M}(\eta, p)\right) \in \bar{J}^{2,+} u(p) .
$$

Here the entries of the (symmetric) $n \times n$ matrix $\mathbf{M}(\eta, p)$ are given by

$$
\mathbf{M}(\eta, p)_{i j}= \begin{cases}\frac{1}{2} \sum_{k=1}^{N} \sum_{l=1}^{N}\left(a_{i l}(p) \frac{\partial a_{j k}}{\partial x_{l}}(p)+a_{j l}(p) \frac{\partial a_{i k}}{\partial x_{l}}(p)\right) \eta_{k}, & i \neq j, \\ \sum_{k=1}^{N} \sum_{l=1}^{N} a_{i l}(p) \frac{\partial a_{i k}}{\partial x_{l}}(p) \eta_{k}, & i=j .\end{cases}
$$

The corollary allows us to "twist" the Euclidean jets into sub-Riemannian jets and enables us to invoke the Euclidean results of [8]. We recall a key theorem.

Theorem 3.3. [8, Theorem 3.2, Remark 3.8] Let $\varepsilon \in \mathbf{R}^{+}$. Let $u$ be an upper semicontinuous function in $\mathbf{R}^{N}, v$ a lower semicontinuous function in $\mathbf{R}^{N}$ and $\phi$ a $C^{2}$ function in $\mathbf{R}^{2 N}$. Let $\mathcal{O}$ be a locally compact subset of $\mathbf{R}^{N}$ and let $(\hat{p}, \hat{q})$ be a maximum point of $u(p)-v(q)-\phi(p, q)$ over $\mathcal{O} \times \mathcal{O}$ and let the matrix $\mathcal{M} \in S^{2 N}$ be given by

$$
\mathcal{M}=\left(\begin{array}{cc}
D_{p p}^{2} \phi(\hat{p}, \hat{q}) & D_{p q}^{2} \phi(\hat{p}, \hat{q}) \\
D_{q p}^{2} \phi(\hat{p}, \hat{q}) & D_{q q}^{2} \phi(\hat{p}, \hat{q})
\end{array}\right) .
$$

Then, there are matrices $X, Y \in S^{N}$ such that

$$
\left(D_{p} \phi(\hat{p}, \hat{q}), X\right) \in \bar{J}_{\text {eucl }}^{2,+} u(\hat{p}) \text { and }\left(-D_{q} \phi(\hat{p}, \hat{q}), Y\right) \in \bar{J}_{\text {eucl }}^{2,-} v(\hat{q}) \text {. }
$$


In addition, for all vectors $\vec{a}, \vec{b} \in \mathbf{R}^{N}$,

$$
\langle X \vec{a}, \vec{a}\rangle-\langle Y \vec{b}, \vec{b}\rangle \leq\left\langle\left(\varepsilon \mathcal{M}^{2}+\mathcal{M}\right)(\vec{a} \oplus \vec{b}),(\vec{a} \oplus \vec{b})\right\rangle
$$

We then combine this result with the twisting to produce the following theorem.

Theorem 3.4. Given a Euclidean $C^{2}$ function $\phi: G \times G \rightarrow \mathbf{R}$, let the semihorizontal gradient at the point $r \in G$ be denoted $\nabla_{1, r} \phi$ and the symmetrized second derivative at $r$ be denoted $\left(D_{r}^{2} \phi\right)^{\star}$. Let $\varepsilon \in \mathbf{R}^{+}$. Let $u, v, \hat{p}, \hat{q}, \mathcal{O}$, and $\mathcal{M}$ be as in Theorem 3.3. Then there are matrices $\mathcal{X}, \mathcal{Y} \in S^{n}$ so that

$$
\left(\nabla_{1, p} \phi(\hat{p}, \hat{q}), \mathcal{X}\right) \in \bar{J}^{2,+} u(\hat{p}) \text { and }\left(-\nabla_{1, q} \phi(\hat{p}, \hat{q}), \mathcal{Y}\right) \in \bar{J}^{2,-} v(\hat{q}) \text {. }
$$

In addition, for all vectors $\xi, \epsilon \in V_{1}$

$$
\langle\mathcal{X} \xi, \xi\rangle-\langle\mathcal{Y} \epsilon, \epsilon\rangle \leq\langle\mathcal{C}(\xi \oplus \epsilon),(\xi \oplus \epsilon)\rangle+\left\langle\varepsilon \mathcal{M}^{2}\left(\mathbf{A}(\hat{p})^{T} \xi \oplus \mathbf{A}(\hat{q})^{T} \epsilon\right),\left(\mathbf{A}(\hat{p})^{T} \xi \oplus \mathbf{A}(\hat{q})^{T} \epsilon\right)\right\rangle .
$$

Here the $2 n \times 2 n$ symmetric matrix $\mathcal{C}$ is given in block form by

$$
\left(\begin{array}{cc}
\left(D_{p}^{2} \phi\right)^{\star}(\hat{p}, \hat{q}) & W \\
W^{T} & \left(D_{q}^{2} \phi\right)^{\star}(\hat{p}, \hat{q})
\end{array}\right) .
$$

and the $n \times n$ matrix $W$ has entries

$$
W_{i j}=X_{i}(p) X_{j}(q) \phi(\hat{p}, \hat{q}) .
$$

Proof. By Theorem 3.3, there are matrices $X$ and $Y$ so that

$$
\left(\nabla_{p} \phi(\hat{p}, \hat{q}), X\right) \in \bar{J}_{\text {eucl }}^{2,+} u(\hat{p}) \text { and }\left(-\nabla_{q} \phi(\hat{p}, \hat{q}), Y\right) \in \bar{J}_{\text {eucl }}^{2,-} v(\hat{q}) \text {. }
$$

By Corollary 3.2, we have

$$
\left(\mathbf{A}(\hat{p}) \cdot \nabla_{p} \phi(\hat{p}, \hat{q}) \oplus \mathbf{B}(\hat{p}) \cdot \nabla_{p} \phi(\hat{p}, \hat{q}), \mathbf{A}(\hat{p}) X \mathbf{A}(\hat{p})^{T}+\mathbf{M}(\hat{p})\right) \in \bar{J}^{2,+} u(\hat{p})
$$

and

$$
\left(-\left(\mathbf{A}(\hat{q}) \cdot \nabla_{q} \phi(\hat{p}, \hat{q}) \oplus \mathbf{B}(\hat{q}) \cdot \nabla_{q} \phi(\hat{p}, \hat{q})\right), \mathbf{A}(\hat{q}) Y \mathbf{A}(\hat{q})^{T}+\mathbf{M}(\hat{q})\right) \in \bar{J}^{2,-} v(\hat{q}) .
$$

By Lemma 3.1, we have, for appropriate matrices $\mathcal{X}$ and $\mathcal{Y}$,

$$
\left(\nabla_{1, p} \phi(\hat{p}, \hat{q}), \mathcal{X}\right) \in \bar{J}^{2,+} u(\hat{p}) \text { and }\left(-\nabla_{1, q} \phi(\hat{p}, \hat{q}), \mathcal{Y}\right) \in \bar{J}^{2,-} v(\hat{q}) .
$$

In addition, untwisting and using Theorem 3.3 produces, for any vectors $\xi, \epsilon \in V_{1}$,

$$
\begin{aligned}
&\langle\mathcal{X} \xi, \xi\rangle-\langle\mathcal{Y} \epsilon, \epsilon\rangle \\
&=\left\langle\left(\mathbf{A}(\hat{p}) X \mathbf{A}(\hat{p})^{T}\right) \xi, \xi\right\rangle+\langle\mathbf{M}(\hat{p}) \xi, \xi\rangle-\left\langle\left(\mathbf{A}(\hat{q}) Y \mathbf{A}(\hat{q})^{T}\right) \epsilon, \epsilon\right\rangle-\langle\mathbf{M}(\hat{q}) \epsilon, \epsilon\rangle \\
&=\left\langle X \mathbf{A}(\hat{p})^{T} \xi, \mathbf{A}(\hat{p})^{T} \xi\right\rangle-\left\langle Y \mathbf{A}(\hat{q})^{T} \epsilon, \mathbf{A}(\hat{q})^{T} \epsilon\right\rangle+\langle\mathbf{M}(\hat{p}) \xi, \xi\rangle-\langle\mathbf{M}(\hat{q}) \epsilon, \epsilon\rangle \\
& \leq\left\langle\left(\varepsilon \mathcal{M}^{2}+\mathcal{M}\right)\left(\mathbf{A}(\hat{p})^{T} \xi \oplus \mathbf{A}(\hat{q})^{T} \epsilon\right),\left(\mathbf{A}(\hat{p})^{T} \xi \oplus \mathbf{A}(\hat{q})^{T} \epsilon\right)\right\rangle+\langle\mathbf{M}(\hat{p}) \xi, \xi\rangle-\langle\mathbf{M}(\hat{q}) \epsilon, \epsilon\rangle \\
&=\left\langle\mathcal{M}\left(\mathbf{A}(\hat{p})^{T} \xi \oplus \mathbf{A}(\hat{q})^{T} \epsilon\right),\left(\mathbf{A}(\hat{p})^{T} \xi \oplus \mathbf{A}(\hat{q})^{T} \epsilon\right)\right\rangle+\langle\mathbf{M}(\hat{p}) \xi, \xi\rangle-\langle\mathbf{M}(\hat{q}) \epsilon, \epsilon\rangle \\
&+\left\langle\varepsilon \mathcal{M}^{2}\left(\mathbf{A}(\hat{p})^{T} \xi \oplus \mathbf{A}(\hat{q})^{T} \epsilon\right),\left(\mathbf{A}(\hat{p})^{T} \xi \oplus \mathbf{A}(\hat{q})^{T} \epsilon\right)\right\rangle .
\end{aligned}
$$

Using the second-order derivative formula of Lemma 3.1 and the formula for $\mathcal{M}$ given in equation (3.2), we have

$$
\begin{aligned}
\left\langle\mathcal{M}\left(\mathbf{A}(\hat{p})^{T} \xi \oplus \mathbf{A}(\hat{q})^{T} \epsilon\right),\left(\mathbf{A}(\hat{p})^{T} \xi \oplus \mathbf{A}(\hat{q})^{T} \epsilon\right)\right\rangle \\
=\left\langle\left(\mathbf{A}(\hat{p}) D_{p p}^{2} \phi(\hat{p}, \hat{q}) \mathbf{A}(\hat{p})^{T}\right) \xi, \xi\right\rangle+\left\langle\left(\mathbf{A}(\hat{p}) D_{p q}^{2} \phi(\hat{p}, \hat{q}) \mathbf{A}(\hat{q})^{T}\right) \epsilon, \xi\right\rangle \\
\quad+\left\langle\left(\mathbf{A}(\hat{q}) D_{q p}^{2} \phi(\hat{p}, \hat{q}) \mathbf{A}(\hat{p})^{T}\right) \xi, \epsilon\right\rangle+\left\langle\left(\mathbf{A}(\hat{q}) D_{q q}^{2} \phi(\hat{p}, \hat{q}) \mathbf{A}(\hat{q})^{T}\right) \epsilon, \epsilon\right\rangle
\end{aligned}
$$


so that

$$
\begin{aligned}
&\langle\mathcal{X} \xi, \xi\rangle-\langle\mathcal{Y} \epsilon, \epsilon\rangle \\
& \leq\left\langle\left(\mathbf{A}(\hat{p}) D_{p p}^{2} \phi(\hat{p}, \hat{q}) \mathbf{A}(\hat{p})^{T}+\mathbf{M}(\hat{p})\right) \xi, \xi\right\rangle+\left\langle\left(\mathbf{A}(\hat{p}) D_{p q}^{2} \phi(\hat{p}, \hat{q}) \mathbf{A}(\hat{q})^{T}\right) \epsilon, \xi\right\rangle \\
&+\left\langle\left(\mathbf{A}(\hat{q}) D_{q p}^{2} \phi(\hat{p}, \hat{q}) \mathbf{A}(\hat{p})^{T}\right) \xi, \epsilon\right\rangle+\left\langle\left(\mathbf{A}(\hat{q}) D_{q q}^{2} \phi(\hat{p}, \hat{q}) \mathbf{A}(\hat{q})^{T}-\mathbf{M}(\hat{q})\right) \epsilon, \epsilon\right\rangle \\
&+\left\langle\varepsilon \mathcal{M}^{2}\left(\mathbf{A}(\hat{p})^{T} \xi \oplus \mathbf{A}(\hat{q})^{T} \epsilon\right),\left(\mathbf{A}(\hat{p})^{T} \xi \oplus \mathbf{A}(\hat{q})^{T} \epsilon\right)\right\rangle .
\end{aligned}
$$

We note that by Lemma 3.2 the " $\eta_{k}$ " term in the definition of $\mathbf{M}(\eta, \hat{q})$ is $-\frac{\partial}{\partial y_{k}} \phi(\hat{p}, \hat{q})$. So that applying Lemma 3.1, we have

$$
\mathbf{A}(\hat{q}) D_{q q}^{2} \phi(\hat{p}, \hat{q}) \mathbf{A}(\hat{q})^{T}-\mathbf{M}(\hat{q})=\left(D_{q}^{2} \phi\right)^{\star}(\hat{p}, \hat{q})
$$

and similarly,

$$
\mathbf{A}(\hat{p}) D_{p p}^{2} \phi(\hat{p}, \hat{q}) \mathbf{A}(\hat{p})^{T}+\mathbf{M}(\hat{p})=\left(D_{p}^{2} \phi\right)^{\star}(\hat{p}, \hat{q}) .
$$

The theorem then follows.

The theorem can be adjusted in the case when $\xi=\epsilon$. Namely,

Corollary 3.5. Let $\varepsilon, \phi, u, v, \hat{p}, \hat{q}, \mathcal{O}$, and $\mathcal{M}$ be as in Theorem 3.4. Then there are matrices $\mathcal{X}, \mathcal{Y} \in S^{n}$ so that

$$
\left(\nabla_{1, p} \phi(\hat{p}, \hat{q}), \mathcal{X}\right) \in \bar{J}^{2,+} u(\hat{p}) \text { and }\left(-\nabla_{1, q} \phi(\hat{p}, \hat{q}), \mathcal{Y}\right) \in \bar{J}^{2,-} v(\hat{q}) .
$$

Additionally, for all vectors $\xi \in V_{1}$

$$
\begin{aligned}
& \langle\mathcal{X} \xi, \xi\rangle-\langle\mathcal{Y} \xi, \xi\rangle \\
& \leq\langle\widetilde{\mathcal{C}}(\xi \oplus \xi),(\xi \oplus \xi)\rangle+\left\langle\varepsilon \mathcal{M}^{2}\left(\mathbf{A}(\hat{p})^{T} \xi \oplus \mathbf{A}(\hat{q})^{T} \xi\right),\left(\mathbf{A}(\hat{p})^{T} \xi \oplus \mathbf{A}(\hat{q})^{T} \xi\right)\right\rangle
\end{aligned}
$$

where the $2 n \times 2 n$ symmetric matrix $\widetilde{\mathcal{C}}$ is given in block form by

$$
\left(\begin{array}{cc}
\left(D_{p}^{2} \phi\right)^{\star}(\hat{p}, \hat{q}) & W^{\star} \\
W^{\star} & \left(D_{q}^{2} \phi\right)^{\star}(\hat{p}, \hat{q})
\end{array}\right)
$$

and the $n \times n$ symmetric matrix $W^{\star}$ has entries

$$
W_{i j}^{\star}=\frac{1}{2}\left(X_{i}(p) X_{j}(q)+X_{j}(p) X_{i}(q)\right) \phi(\hat{p}, \hat{q}) .
$$

Proof. Define the $2 n \times 2 n$ matrix

$$
\mathfrak{M}=\left(\begin{array}{cc}
0 & \frac{1}{2}\left(W-W^{T}\right) \\
\frac{1}{2}\left(W^{T}-W\right) & 0
\end{array}\right)
$$

and note that for any vector $\xi \in V_{1}$, we have

$$
\langle\mathfrak{M}(\xi \oplus \xi),(\xi \oplus \xi)\rangle=0 .
$$

Invoking the theorem, we have

$$
\left(\nabla_{1, p} \phi(\hat{p}, \hat{q}), \mathcal{X}\right) \in \bar{J}^{2,+} u(\hat{p}) \text { and }\left(-\nabla_{1, q} \phi(\hat{p}, \hat{q}), \mathcal{Y}\right) \in \bar{J}^{2,-} v(\hat{q})
$$

with

$$
\langle\mathcal{X} \xi, \xi\rangle-\langle\mathcal{Y} \xi, \xi\rangle \leq\langle\mathcal{C}(\xi \oplus \xi),(\xi \oplus \xi)\rangle+\left\langle\varepsilon \mathcal{M}^{2}\left(\mathbf{A}(\hat{p})^{T} \xi \oplus \mathbf{A}(\hat{q})^{T} \xi\right),\left(\mathbf{A}(\hat{p})^{T} \xi \oplus \mathbf{A}(\hat{q})^{T} \xi\right)\right\rangle .
$$

Recall that

$$
\mathcal{C}=\left(\begin{array}{cc}
\left(D_{p}^{2} \phi\right)^{\star}(\hat{p}, \hat{q}) & W \\
W^{T} & \left(D_{q}^{2} \phi\right)^{\star}(\hat{p}, \hat{q})
\end{array}\right) .
$$

We then observe that $\mathcal{C}=\widetilde{\mathcal{C}}+\mathfrak{M}$. The result then follows from equation (3.4). 
Theorem 3.4 and Corollary 3.5 hold in any sub-Riemannian space. However, when using them to prove comparison principles in Carnot groups, they produce estimates that are not readily controlled. This is not unexpected, as we have not used the fact that we are in a Carnot group. By incorporating the Carnot group law with Theorem 3.4, we obtain the Carnot group maximum principle.

Lemma 3.6. (Carnot group maximum principle) Let $\Omega \subset G$ be a domain. Let $\tau \in \mathbf{R}^{+}$and let $u$ be an upper semicontinuous function and $v$ a lower semicontinuous function. Let $\phi(p, q)=\varphi\left(p \cdot q^{-1}\right)$ be a non-negative $C^{2}$ function so that $\varphi\left(p \cdot q^{-1}\right)=0$ exactly when $p=q$. Let the points $p_{\tau}, q_{\tau} \in G$ be the local maximum in $\Omega \times \Omega$ of $u(p)-v(q)-\tau \varphi\left(p \cdot q^{-1}\right)$ and let $u-v$ have a positive interior local maximum

$$
\sup _{\Omega}(u-v)>0 .
$$

The following hold:

i)

$$
\lim _{\tau \rightarrow \infty} \tau \varphi\left(p_{\tau} \cdot q_{\tau}^{-1}\right)=0
$$

ii) There exists a point $\hat{p} \in \Omega$ such that $p_{\tau} \rightarrow \hat{p}$ (and so does $q_{\tau}$ by (i)) and

$$
\sup _{\Omega}(u-v)=u(\hat{p})-v(\hat{p})>0 .
$$

iii) There exist symmetric matrices $\mathcal{X}_{\tau}, \mathcal{Y}_{\tau}$ and vector $\eta_{\tau} \in V_{1} \oplus V_{2}$, namely $\eta_{\tau}=\nabla_{1, p} \varphi\left(p_{\tau} \cdot q_{\tau}^{-1}\right)$, so that

$$
\left(\tau \eta_{\tau}, \mathcal{X}_{\tau}\right) \in \bar{J}^{2,+} u\left(p_{\tau}\right) \text { and }\left(\tau \eta_{\tau}, \mathcal{Y}_{\tau}\right) \in \bar{J}^{2,-} v\left(q_{\tau}\right) .
$$

vi) For any vectors $\xi, \epsilon \in V_{1}$, we have

$$
\begin{aligned}
& \left\langle\mathcal{X}_{\tau} \xi, \xi\right\rangle-\left\langle\mathcal{Y}_{\tau} \epsilon, \epsilon\right\rangle \\
& \leq \tau\left\langle\left(D_{p}^{2} \varphi\right)^{\star}\left(p_{\tau} \cdot q_{\tau}^{-1}\right)(\xi-\epsilon),(\xi-\epsilon)\right\rangle+\tau\langle\mathfrak{M}(\xi \oplus \epsilon),(\xi \oplus \epsilon)\rangle \\
& \quad+\tau\|\mathcal{M}\|^{2}\left\|\mathbf{A}(\hat{p})^{T} \xi \oplus \mathbf{A}(\hat{q})^{T} \epsilon\right\|^{2} .
\end{aligned}
$$

In particular,

$$
\left\langle\mathcal{X}_{\tau} \xi, \xi\right\rangle-\left\langle\mathcal{Y}_{\tau} \xi, \xi\right\rangle \lesssim \tau\|\mathcal{M}\|^{2}\|\xi\|^{2}
$$

Recall $\mathcal{M}$ is the matrix defined in equation (3.2) and $\mathfrak{M}$ is the matrix defined in equation (3.3).

Proof. The proof of the first two items follows that of the Euclidean case [8] and Heisenberg group case [3] and is omitted. In order to prove the last two items, we note that

$$
\begin{aligned}
X_{j}(p) \varphi\left(p \cdot q^{-1}\right) & =\left.\frac{d}{d t} \varphi\left(p \cdot \exp \left(t X_{j}\right) \cdot q^{-1}\right)\right|_{t=0} \\
& =\left.\frac{d}{d t} \varphi\left(p \cdot\left(q \cdot \exp \left(-t X_{j}\right)\right)^{-1}\right)\right|_{t=0} \\
& =-X_{j}(q) \varphi\left(p \cdot q^{-1}\right)
\end{aligned}
$$

with a similar calculation holding for $Y_{j}(p)$. Consequently, we have

$$
\left(X_{i}(p) X_{j}(q)+X_{j}(p) X_{i}(q)\right) \varphi\left(p \cdot q^{-1}\right)=-\left(X_{i}(p) X_{j}(p)+X_{j}(p) X_{i}(p)\right) \varphi\left(p \cdot q^{-1}\right)
$$


In a similar manner, we also have

$$
\begin{aligned}
& \frac{d}{d s} \frac{d}{d t} \varphi\left(p \cdot \exp \left(t X_{j}\right) \cdot \exp \left(s X_{i}\right) \cdot q^{-1}\right)+\frac{d}{d t} \frac{d}{d s} \varphi\left(p \cdot \exp \left(s X_{i}\right) \cdot \exp \left(t X_{j}\right) \cdot q^{-1}\right) \\
& =\frac{d}{d s} \frac{d}{d t} \varphi\left(p \cdot\left(q \cdot \exp \left(-s X_{i}\right) \cdot \exp \left(-t X_{j}\right)\right)^{-1}\right) \\
& \quad+\frac{d}{d t} \frac{d}{d s} \varphi\left(p \cdot\left(q \cdot \exp \left(-t X_{j}\right) \cdot \exp \left(-s X_{i}\right)\right)^{-1}\right)
\end{aligned}
$$

allowing us to conclude

$$
\begin{aligned}
& \left(X_{j}(p) X_{i}(p)+X_{i}(p) X_{j}(p)\right) \varphi\left(p \cdot q^{-1}\right) \\
& =(-1)^{2}\left(X_{j}(q) X_{i}(q)+X_{i}(q) X_{j}(q)\right) \varphi\left(p \cdot q^{-1}\right) .
\end{aligned}
$$

Set $\eta_{\tau}=\nabla_{1, p} \varphi\left(p_{\tau} \cdot q_{\tau}^{-1}\right)=-\nabla_{1, q} \varphi\left(p_{\tau} \cdot q_{\tau}^{-1}\right)$. Then Corollary 3.2 produces

$$
\left(\tau \eta_{\tau}, \mathcal{X}_{\tau}\right) \in \bar{J}^{2,+} u\left(p_{\tau}\right) \text { and }\left(\tau \eta_{\tau}, \mathcal{Y}_{\tau}\right) \in \bar{J}^{2,-} v\left(q_{\tau}\right)
$$

for appropriate matrices $\mathcal{X}_{\tau}$ and $\mathcal{Y}_{\tau}$, proving the third item.

In addition, Corollary 3.5 and equations (3.7) and (3.8) produce, for any vectors $\xi, \epsilon \in V_{1}$,

$$
\begin{aligned}
\left\langle\mathcal{X}_{\tau} \xi, \xi\right\rangle-\left\langle\mathcal{Y}_{\tau} \epsilon, \epsilon\right\rangle \leq & \tau\left\langle\left(\begin{array}{cc}
\left(D_{p}^{2} \varphi\right)^{\star}\left(p_{\tau} \cdot q_{\tau}^{-1}\right) & -\left(D_{p}^{2} \varphi\right)^{\star}\left(p_{\tau} \cdot q_{\tau}^{-1}\right) \\
-\left(D_{p}^{2} \varphi\right)^{\star}\left(p_{\tau} \cdot q_{\tau}^{-1}\right) & \left(D_{p}^{2} \varphi\right)^{\star}\left(p_{\tau} \cdot q_{\tau}^{-1}\right)
\end{array}\right)\left(\begin{array}{l}
\xi \\
\epsilon
\end{array}\right),\left(\begin{array}{l}
\xi \\
\epsilon
\end{array}\right)\right\rangle \\
& +\tau\langle\mathfrak{M}(\xi \oplus \epsilon),(\xi \oplus \epsilon)\rangle \\
& +\tau^{2}\left\langle\varepsilon \mathcal{M}^{2}\left(\mathbf{A}(\hat{p})^{T} \xi \oplus \mathbf{A}(\hat{q})^{T} \epsilon\right),\left(\mathbf{A}(\hat{p})^{T} \xi \oplus \mathbf{A}(\hat{q})^{T} \epsilon\right)\right\rangle .
\end{aligned}
$$

Equation (3.5) follows by setting $\varepsilon=\tau^{-1}$ and equation (3.6) follows from equation (3.5) by setting $\epsilon=\xi$.

\section{Infinite harmonic functions}

The existence of viscosity infinite harmonic functions in Carnot groups can be proved by following the Heisenberg argument in [3]. In [15], Wang uses the clever technique of considering the Carnot infinite Laplace equation from the Euclidean point of view to obtain uniqueness of viscosity solutions. The key step is to show uniqueness of viscosity solutions to the Jensen auxiliary functions [12] and pass to the limit. We will present a proof of this key step from the sub-Riemannian point of view by using the Carnot group maximum principle. Namely,

Theorem 4.1. Fix $\varepsilon>0$. Define the function $F_{\infty}^{\varepsilon}$ by

$$
F_{\infty}^{\varepsilon}\left(\nabla_{1} u,\left(D^{2} u\right)^{\star}\right)=\min \left\{\left\|\nabla_{0} u\right\|^{2}-\varepsilon,-\left\langle\left(D^{2} u\right)^{\star} \nabla_{0} u, \nabla_{0} u\right\rangle\right\} .
$$

Let $u$ be a viscosity subsolution and $v$ a viscosity supersolution to $F_{\infty}^{\varepsilon}=0$ in a bounded domain $\Omega$. Then

$$
\sup _{p \in \bar{\Omega}}(u(p)-v(p))=\sup _{p \in \partial \Omega}(u(p)-v(p)) .
$$

Proof. Suppose not. Then,

$$
\sup _{p \in \bar{\Omega}}(u(p)-v(p))>\sup _{p \in \partial \Omega}(u(p)-v(p)) .
$$


Using Lemma 5.1 from [3], we may replace $v$ with the strict supersolution $w$, so that $F_{\infty}^{\varepsilon}\left(\left(\nabla_{1} w,\left(D^{2} w\right)^{\star}\right)>0\right.$. Consider the function

$$
\varphi\left(p \cdot q^{-1}\right)=\sum_{i=1}^{N} \frac{1}{6}\left(\left(p \cdot q^{-1}\right)_{i}\right)^{6}
$$

where $\left(p \cdot q^{-1}\right)_{i}$ is the $i$-th component of the group multiplication law. We let the maximum of $u(p)-v(q)-\tau \varphi\left(p \cdot q^{-1}\right)$ in $\bar{\Omega} \times \bar{\Omega}$ occur at the point $\left(p_{\tau}, q_{\tau}\right)$. Applying the chain rule, we have

$$
\begin{aligned}
\frac{\partial}{\partial x_{j}} \varphi\left(p_{\tau} \cdot q_{\tau}^{-1}\right)= & \sum_{i=1}^{N}\left(\left(p_{\tau} \cdot q_{\tau}^{-1}\right)_{i}\right)^{5} \frac{\partial}{\partial x_{j}}\left(p_{\tau} \cdot q_{\tau}^{-1}\right)_{i}, \\
\frac{\partial}{\partial x_{j}} \frac{\partial}{\partial x_{k}} \varphi\left(p_{\tau} \cdot q_{\tau}^{-1}\right)= & \sum_{i=1}^{N}\left(\left(p_{\tau} \cdot q_{\tau}^{-1}\right)_{i}\right)^{5} \frac{\partial}{\partial x_{j}} \frac{\partial}{\partial x_{k}}\left(p_{\tau} \cdot q_{\tau}^{-1}\right)_{i} \\
& +\sum_{i=1}^{N} 5\left(\left(p_{\tau} \cdot q_{\tau}^{-1}\right)_{i}\right)^{4}\left(\frac{\partial}{\partial x_{j}}\left(p_{\tau} \cdot q_{\tau}^{-1}\right)_{i}\right)\left(\frac{\partial}{\partial x_{k}}\left(p_{\tau} \cdot q_{\tau}^{-1}\right)_{i}\right)
\end{aligned}
$$

with corresponding formulas for $\frac{\partial}{\partial y_{j}} \varphi\left(p_{\tau} \cdot q_{\tau}^{-1}\right)$ and $\frac{\partial}{\partial y_{j}} \frac{\partial}{\partial y_{k}} \varphi\left(p_{\tau} \cdot q_{\tau}^{-1}\right)$. We note that $\frac{\partial}{\partial x_{j}}\left(p_{\tau} \cdot q_{\tau}^{-1}\right)_{i}$ is a polynomial in $p_{\tau}$ and $q_{\tau}$, which are points in a bounded domain. We therefore can find a constant $K<\infty$ so that for all $l$ and $i$,

$$
\left|\frac{\partial}{\partial x_{l}}\left(p_{\tau} \cdot q_{\tau}^{-1}\right)_{i}\right| \leq K \text { and }\left|\frac{\partial}{\partial y_{l}}\left(p_{\tau} \cdot q_{\tau}^{-1}\right)_{i}\right| \leq K
$$

We then conclude

$$
\left|\frac{\partial}{\partial x_{j}} \varphi\left(p_{\tau} \cdot q_{\tau}^{-1}\right)\right| \lesssim \varphi\left(p_{\tau} \cdot q_{\tau}^{-1}\right)^{\frac{5}{6}} \text { and }\left|\frac{\partial}{\partial y_{j}} \varphi\left(p_{\tau} \cdot q_{\tau}^{-1}\right)\right| \lesssim \varphi\left(p_{\tau} \cdot q_{\tau}^{-1}\right)^{\frac{5}{6}} .
$$

Invoking Lemma 3.6 (i) and (ii), namely, that $p_{\tau} \rightarrow \hat{p}$ and $q_{\tau} \rightarrow \hat{p}$, we also have for sufficiently large $\tau$ (so that $\left|p_{\tau} \cdot q_{\tau}^{-1}\right|<1$ ),

$$
\left|\frac{\partial}{\partial x_{j}} \frac{\partial}{\partial x_{k}} \varphi\left(p_{\tau} \cdot q_{\tau}^{-1}\right)\right| \lesssim \varphi\left(p_{\tau} \cdot q_{\tau}^{-1}\right)^{\frac{4}{6}} \text { and }\left|\frac{\partial}{\partial y_{j}} \frac{\partial}{\partial y_{k}} \varphi\left(p_{\tau} \cdot q_{\tau}^{-1}\right)\right| \lesssim \varphi\left(p_{\tau} \cdot q_{\tau}^{-1}\right)^{\frac{4}{6}}
$$

We note that equation (4.2) also holds for the mixed derivatives $\frac{\partial}{\partial x_{j}} \frac{\partial}{\partial y_{k}}$ and $\frac{\partial}{\partial y_{j}} \frac{\partial}{\partial x_{k}}$. By Lemma 3.6, we have

$$
\left(\tau \eta_{\tau}, \mathcal{X}_{\tau}\right) \in \bar{J}^{2,+} u\left(p_{\tau}\right) \text { and }\left(\tau \eta_{\tau}, \mathcal{Y}_{\tau}\right) \in \bar{J}^{2,-} w\left(q_{\tau}\right)
$$

with the matrices satisfying

$$
\left\langle\mathcal{X}_{\tau} \tau \eta_{\tau}, \tau \eta_{\tau}\right\rangle-\left\langle\mathcal{Y}_{\tau} \tau \eta_{\tau}, \tau \eta_{\tau}\right\rangle \leq \tau^{3}\|\mathcal{M}\|^{2}\left\|\eta_{\tau}\right\|^{2}
$$

By the definition of $\mathcal{M}$ (equation (3.2)), and equation (4.2), we have

$$
\|\mathcal{M}\| \lesssim \varphi\left(p_{\tau} \cdot q_{\tau}^{-1}\right)^{\frac{4}{6}} .
$$

In addition, since $\eta_{\tau}=\nabla_{1, p} \varphi\left(p_{\tau} \cdot q_{\tau}^{-1}\right)$, equation (4.1) gives

$$
\|\eta\| \lesssim \varphi\left(p_{\tau} \cdot q_{\tau}^{-1}\right)^{\frac{5}{6}}
$$


We then have

$$
\begin{aligned}
\left\langle\mathcal{X}_{\tau} \tau \eta_{\tau}, \tau \eta_{\tau}\right\rangle-\left\langle\mathcal{Y}_{\tau} \tau \eta_{\tau}, \tau \eta_{\tau}\right\rangle & \leq \tau^{3}\|\mathcal{M}\|^{2}\left\|\eta_{\tau}\right\|^{2} \\
& \lesssim \tau^{3} \varphi\left(p \cdot q^{-1}\right)^{\frac{4}{3}} \times \varphi\left(p \cdot q^{-1}\right)^{\frac{5}{3}}=\left(\tau \varphi\left(p \cdot q^{-1}\right)\right)^{3} .
\end{aligned}
$$

Now, since $u$ is a subsolution and $w$ a strict supersolution, we have

$$
\min \left\{\left\|\tau \eta_{\tau}\right\|^{2}-\varepsilon,-\left\langle\mathcal{X}_{\tau} \tau \eta_{\tau}, \tau \eta_{\tau}\right\rangle\right\} \leq 0
$$

and

$$
\min \left\{\left\|\tau \eta_{\tau}\right\|^{2}-\varepsilon,-\left\langle\mathcal{Y}_{\tau} \tau \eta_{\tau}, \tau \eta_{\tau}\right\rangle\right\}>0
$$

so that subtracting, we have

$$
\begin{aligned}
0 & <\min \left\{0,\left\langle\mathcal{X}_{\tau} \tau \eta_{\tau}, \tau \eta_{\tau}\right\rangle-\left\langle\mathcal{Y}_{\tau} \tau \eta_{\tau}, \tau \eta_{\tau}\right\rangle\right\} \\
& \lesssim\left(\tau \phi\left(p \cdot q^{-1}\right)\right)^{3} \rightarrow 0 \quad \text { as } \quad \tau \rightarrow \infty
\end{aligned}
$$

The theorem follows from this contradiction.

Uniqueness of viscosity infinite harmonic functions then follows as in [3].

\section{The $p$-Laplacian in Carnot groups}

We now turn our attention to the $p$-Laplacian when $1<p<\infty$. Our main goal is to relate three different notions of solutions to the equation

$$
-\Delta_{p} f=-\operatorname{div}\left(\left\|\nabla_{0} f\right\|^{p-2} \nabla_{0} f\right)=0
$$

in a bounded domain $\Omega$.

5.1. Weak solutions. We begin by considering weak solutions to equation (5.1). We will actually do more, for we shall consider weak solutions to a wider class of equations. Letting $\varepsilon \geq 0$ be a real parameter, we consider equations of the form

$$
-\Delta_{p} f=-\operatorname{div}\left(\left\|\nabla_{0} f\right\|^{p-2} \nabla_{0} f\right)=\varepsilon
$$

in a bounded domain $\Omega$. Note that equation (5.1) corresponds to equation (5.2) with $\varepsilon=0$. We define weak solutions to equation (5.1) and $\varepsilon$-weak solutions to equation (5.2) in the usual way. A weak solution to equation (5.1) (i.e., a 0-weak solution) is called p-harmonic.

In addition to weak solutions we may define weak supersolutions and weak subsolutions in the usual way. Using the definitions for $\varepsilon_{1}>\varepsilon_{2} \geq 0$, we observe that an $\varepsilon_{1}$-weak solution is a $\varepsilon_{2}$-weak supersolution and an $\varepsilon_{2}$-weak solution is a $\varepsilon_{1}$-weak subsolution.

It is also well-known that 0 -weak subsolutions and supersolutions satisfy the following comparison principle.

Lemma 5.1. [11, Lemma 3.18] Let $u \in W^{1, p}(\Omega)$ be a weak subsolution to equation (5.1) and let $v \in W^{1, p}(\Omega)$ be a weak supersolution to equation (5.1) in $\Omega$. If $\gamma \equiv \min \{v-u, 0\} \in W_{0}^{1, p}(\Omega)$, then $u \leq v$ almost everywhere in $\Omega$.

We are then able to formulate the existence-uniqueness of $p$-harmonic functions. (cf. [11, Thm. 3.17], [10, Sec. 4.10]).

Theorem 5.2. Given a bounded domain $\Omega$ with boundary data $\Theta \in W^{1, p}(\Omega)$, there is a unique $p$-harmonic function $u$ that satisfies $u-\Theta \in W_{0}^{1, p}(\Omega)$. 
Using standard techniques in calculus of variations, one can show that $\varepsilon$-weak solutions exist and Lemma 5.1 can be extended to $\varepsilon$-weak solutions.

It is well-known that an $\varepsilon$-weak solution $u$ has a continuous representative that satisfies

$$
\operatorname{osc}_{B_{r}} u \leq C_{\varepsilon}\left(\frac{r}{R}\right)^{\alpha_{\varepsilon}} \operatorname{osc}_{B_{R}}
$$

when $B_{R} \subset \Omega$ and $r \leq R$. ([10,6]) We note that the constants $C_{\varepsilon}>0$ and $\alpha_{\varepsilon}>0$ depend only on $\varepsilon$ and the group $G$. We therefore identify $\varepsilon$-weak solutions with their continuous representative.

5.2. $p$-superharmonic functions. The next class of solutions we wish to consider are $p$-superharmonic functions and $p$-subharmonic functions defined via the following definition.

Definition 2. The function $u: \Omega \rightarrow \mathbf{R}^{N} \cup\{\infty\}$ is p-superharmonic if the following hold:

(1) $u$ is lower semicontinuous.

(2) $u$ is not identically infinity in each component of $\Omega$.

(3) For each subdomain $D \subset \subset \Omega$, a $p$-harmonic function $g$ in $D$ that is continuous in $\bar{D}$ with $g \leq u$ on $\partial D$ implies $g \leq u$ in $D$.

A function $u$ is $p$-subharmonic if $-u$ is $p$-superharmonic.

These key point of these definitions are that they are based on comparison with $p$-harmonic functions. We then are able to obtain the following comparison principle [14, Thm. 7.2].

Lemma 5.3. Let $\Omega$ be a bounded domain in $G$. Let $v$ be a $p$-superharmonic function and $u$ be a $p$-subharmonic function in $\Omega$ so that

$$
\limsup _{q \rightarrow p} u(q) \leq \liminf _{q \rightarrow p} v(q)
$$

for all $p \in \partial \Omega$ with both sides not simultaneously $-\infty$ or $\infty$. Then $u \leq v$ in $\Omega$.

We are then able to conclude the following lemma ([11, Lemma 7.8]).

Lemma 5.4. A function is $p$-harmonic if and only if it is both p-subharmonic and $p$-superharmonic.

5.3. Viscosity solutions. We consider equation (5.2) in non-divergence form, namely,

$$
-\left(\left\|\nabla_{0} u\right\|^{p-2} \operatorname{tr}\left(\left(D^{2} u\right)^{\star}\right)+(p-2)\left\|\nabla_{0} u\right\|^{p-4}\left\langle\left(D^{2} u\right)^{\star} \nabla_{0} u, \nabla_{0} u\right\rangle\right)=\varepsilon .
$$

We note that equation (5.3) is degenerate elliptic and proper in the sense of [8]. In order to consider the possibility that for a test function $\phi$, we may have $\left\|\nabla_{0} \phi\right\|=0$, resulting in a singularity when $1<p<2$, we will need to weaken the definition of viscosity solution. This definition coincides with the definition given earlier when $2 \leq p<\infty$. (See also [13] and [4] for a further discussion.)

Definition 3. The function $u: \Omega \rightarrow \mathbf{R}^{N} \cup\{\infty\}$ is an $\varepsilon$-viscosity supersolution to equation (5.3) if the following hold:

(1) $u$ is lower semicontinuous.

(2) $u$ is not identically infinity in each component of $\Omega$. 
(3) For $p_{0} \in \Omega, \phi \in \mathcal{T} \mathcal{B}\left(u, p_{0}\right)$ with $\nabla_{0} \phi\left(p_{0}\right) \neq 0$ satisfies

$$
-\Delta_{p} \phi\left(p_{0}\right) \geq \varepsilon \text {. }
$$

A function $u$ is an $\varepsilon$-viscosity subsolution to equation (5.3) if $-u$ is an $\varepsilon$-viscosity supersolution. A function $u$ is an $\varepsilon$-viscosity solution if it is both an $\varepsilon$-viscosity supersolution and an $\varepsilon$-viscosity subsolution.

Given the viscosity solutions, it is natural to ask how they relate to the previous notions of solutions. It was shown via Lemma 4.1 in [3] that upper(lower) semicontinuous $\varepsilon$-weak sub(super-)solutions are $\varepsilon$-viscosity sub(super-)solutions in the Heisenberg group. The proof trivially extends to Carnot groups. In addition, we have the following lemma which was proven for the Heisenberg group in [4], as Lemma 3.5. The proof is similar and omitted.

Lemma 5.5. A p-sub(super-)harmonic function is a viscosity sub(super-)solution. Hence, a p-harmonic function is a viscosity solution.

We now have existence of all three notions of solutions, but a comparison principle only for the first two. We will now establish a comparison principle for viscosity solutions.

We begin with a technical lemma whose Euclidean version is Lemma 3.2 in [13] and Heisenberg version is Lemma 4.1 in [4]. The proof is similar, needing only to use the smooth gauge $\mathcal{N}(p)$, and is therefore omitted.

Lemma 5.6. Let $v \in W_{\text {loc }}^{1, p}$ be a continuous $\varepsilon$-weak solution. Let $p_{0} \in \Omega$ and let $\phi \in \mathcal{C}_{\text {sub }}^{2}(\Omega)$ be a function such that $v-\phi$ has a strict local minimum at $p_{0}$. Then

$$
\limsup _{\substack{p \rightarrow p_{0} \\ p \neq p_{0}}}\left(-\Delta_{p} \phi(p)\right) \geq \varepsilon
$$

provided that $\nabla_{0} \phi\left(p_{0}\right) \neq 0$ or $p_{0}$ is an isolated critical point.

Note that in the case when $p \geq 2$, by continuity we have $-\Delta_{p} \phi\left(p_{0}\right) \geq \varepsilon$ and so $\nabla_{0} \phi(p) \neq 0$ near $p_{0}$.

We next consider the function $\varphi: G \times G \rightarrow \mathbf{R}$ given by

$$
\varphi\left(p \cdot q^{-1}\right)=\frac{1}{m} \sum_{i=1}^{N}\left|\left(p \cdot q^{-1}\right)_{i}\right|^{m}
$$

for some large positive integer $m>4$. We note that 4 is chosen so that $\varphi$ is $\mathcal{C}_{\text {sub }}^{2}$. Here, as before, $\left(p \cdot q^{-1}\right)_{i}$ is the $i$-th component of $p \cdot q^{-1}$.

We now prove a preliminary comparison principle.

Lemma 5.7. Fix $\varepsilon>0$ and $1<p<\infty$. Let $v$ be a continuous $\varepsilon$-weak solution and let $u$ be a 0 -viscosity subsolution so that $u \leq v$ on $\partial \Omega$. Then $u \leq v$ in $\Omega$.

Proof. Suppose that $\sup (u-v)>0$ occurs at the interior point $p_{0}$. For each positive integer $j$, we consider the function $\psi_{j}: G \times G \rightarrow \mathbf{R}$ defined by

$$
\psi_{j}(p, q)=u(p)-v(q)-j \varphi(p, q)
$$

with $m$ chosen so that $m>\max \left\{4, \frac{p}{p-1}, p+2\right\}$. Following the scheme of [3] and [8], we let the maximum of $\psi_{j}$ occur at $\left(p_{j}, q_{j}\right)$ and observe for large $j$, these are interior points. By Lemma 3.6, these points tend to $p_{0}$ as $j \rightarrow \infty$ and

$$
\left(j \eta_{j}, \mathcal{X}_{j}\right) \in \bar{J}^{2,+} u\left(p_{j}\right) \text { and }\left(j \eta_{j}, \mathcal{Y}_{j}\right) \in \bar{J}^{2,-} v\left(q_{j}\right)
$$


where $\eta_{j}, \mathcal{X}$, and $\mathcal{Y}$ are as in Lemma 3.6.

Claim 5.8. By passing to a subsequence if needed, we may assume $p_{j} \neq q_{j}$.

Proof. Fix $j>0$. By definition, we have for any $p$ and $q$,

$$
u(p)-v(q)-j \varphi(p, q) \leq u\left(p_{j}\right)-v\left(q_{j}\right)-j \varphi\left(p_{j}, q_{j}\right)
$$

and so when $p=p_{j}$, we have

$$
v(q) \geq v\left(q_{j}\right)+j \varphi\left(p_{j}, q_{j}\right)-j \varphi\left(p_{j}, q\right) .
$$

Defining the function $\beta(q)$ by

$$
\beta(q)=v\left(q_{j}\right)+j \varphi\left(p_{j}, q_{j}\right)-j \varphi\left(p_{j}, q\right)-\varphi\left(q_{j}, q\right)
$$

we see that $v-\beta$ has a strict local minimum at $q_{j}$ and $q_{j}$ is an isolated critical point. Applying Lemma 5.6, we have

$$
\limsup _{q \rightarrow q_{j}}\left(-\Delta_{p} \beta(q)\right) \geq \varepsilon
$$

Suppose now that $p_{j}=q_{j}$. Then $\beta(q)=v\left(q_{j}\right)-(j+1) \varphi\left(q_{j}, q\right)$. We then need to estimate $\Delta_{p} \beta(q)$. Using the non-divergence form of the $p$-Laplacian (equation (5.3)) and the definition of $\beta(q)$, we have

$$
\left|\Delta_{p} \beta(q)\right| \lesssim\left\|\nabla_{0} \varphi\left(q_{j}, q\right)\right\|^{p-2}\left|\operatorname{tr}\left(D^{2} \varphi\right)^{\star}\left(q_{j}, q\right)+\left\|\left(D^{2} \varphi\right)^{\star}\left(q_{j}, q\right)\right\|\right| .
$$

Using Lemma 3.6, we have

$$
\left\|\nabla_{0} \varphi\left(q_{j}, q\right)\right\| \sim\left\|\eta_{j}\right\| \sim \varphi\left(q_{j}, q\right)^{\frac{m-1}{m}} .
$$

We note that given the standard vectors $e_{k}$ with every entry 0 except for the $k$-th entry which is equal to 1 , we see that for any matrix $A$,

$$
\operatorname{tr}(A)=\sum\left\langle A e_{k}, e_{k}\right\rangle
$$

and so

$$
\left|\operatorname{tr}\left(D^{2} \varphi\right)^{\star}\left(q_{j}, q\right)\right| \lesssim\left\|\left(D^{2} \varphi\right)^{\star}\left(q_{j}, q\right)\right\|
$$

We then conclude

$$
\left|\operatorname{tr}\left(D^{2} \varphi\right)^{\star}\left(q_{j}, q\right)+\left\|\left(D^{2} \varphi\right)^{\star}\left(q_{j}, q\right)\right\|\right| \lesssim \varphi\left(q_{j}, q\right)^{\frac{m-2}{m}}
$$

so that

$$
\left|\Delta_{p} \beta(q)\right| \lesssim\left(\varphi\left(q_{j}, q\right)^{\frac{1}{m}}\right)^{(m-1)(p-2)+(m-2)} .
$$

Since $m>\frac{p}{p-1}$, we would have

$$
\lim _{\substack{q \rightarrow q_{j} \\ q \neq q_{j}}}\left(-\Delta_{p} \beta(q)\right)=0
$$

This contradicts equation (5.4).

Now, $u$ is a viscosity subsolution to equation (5.3) with $\varepsilon=0$. That is,

$$
0 \geq-\left(\left\|j \eta_{j}\left(p_{j}, q_{j}\right)\right\|^{p-2} \operatorname{tr}\left(\mathcal{X}_{j}\right)^{\star}+(p-2)\left\|j \eta_{j}\left(p_{j}, q_{j}\right)\right\|^{p-4}\left\langle\mathcal{X}_{j} j \eta_{j}\left(p_{j}, q_{j}\right), j \eta_{j}\left(p_{j}, q_{j}\right)\right\rangle\right) \text {. }
$$


Using Lemmas 5.5 and 5.6 along with the definition of $\bar{J}^{2,-}$, we have $\varepsilon \leq-\left(\left\|j \eta_{j}\left(p_{j}, q_{j}\right)\right\|^{p-2} \operatorname{tr}\left(\mathcal{Y}_{j}\right)^{\star}+(p-2)\left\|j \eta_{j}\left(p_{j}, q_{j}\right)\right\|^{p-4}\left\langle\mathcal{Y}_{j} j \eta_{j}\left(p_{j}, q_{j}\right), j \eta_{j}\left(p_{j}, q_{j}\right)\right\rangle\right)$.

Subtracting these two inequalities, we have

$$
\begin{aligned}
0<\varepsilon< & j^{p-2}\left\|\eta_{j}\left(p_{j}, q_{j}\right)\right\|^{p-2}\left(\operatorname{tr}\left(\mathcal{X}_{j}\right)-\operatorname{tr}\left(\mathcal{Y}_{j}\right)\right) \\
& +(p-2) j^{p-2}\left\|\eta_{j}\left(p_{j}, q_{j}\right)\right\|^{p-4}\left(\left\langle\mathcal{X}_{j} \eta_{j}\left(p_{j}, q_{j}\right), \eta_{j}\left(p_{j}, q_{j}\right)\right\rangle\right. \\
& \left.-\left\langle\mathcal{Y}_{j} \eta_{j}\left(p_{j}, q_{j}\right), \eta_{j}\left(p_{j}, q_{j}\right)\right\rangle\right) .
\end{aligned}
$$

As in the proof of the above claim, we have

$$
\left\|\eta_{j}\left(p_{j}, q_{j}\right)\right\| \sim \varphi\left(p_{j}, q_{j}\right)^{\frac{m-1}{m}}
$$

and we can write the trace difference as

$$
\operatorname{tr}\left(\mathcal{X}_{j}\right)-\operatorname{tr}\left(\mathcal{Y}_{j}\right)=\sum_{k=1}^{2 n}\left\langle\mathcal{X}_{j} e_{k}, e_{k}\right\rangle-\left\langle\mathcal{Y}_{j} e_{k}, e_{k}\right\rangle .
$$

Using Lemma 3.6, we obtain

$$
\operatorname{tr}\left(\mathcal{X}_{j}\right)-\operatorname{tr}\left(\mathcal{Y}_{j}\right) \lesssim j\left(\varphi\left(p_{j}, q_{j}\right)^{\frac{2 m-4}{m}}\right)
$$

and

$$
\left\langle\mathcal{X}_{j} \eta_{j}\left(p_{j}, q_{j}\right), \eta_{j}\left(p_{j}, q_{j}\right)\right\rangle-\left\langle\mathcal{Y}_{j} \eta_{j}\left(p_{j}, q_{j}\right), \eta_{j}\left(p_{j}, q_{j}\right)\right\rangle \lesssim j\left(\varphi\left(p_{j}, q_{j}\right)^{\frac{2 m-4}{m}}\right)\left(\varphi\left(p_{j}, q_{j}\right)^{\frac{2 m-2}{m}}\right) .
$$

Equation (5.5) then leads to

$$
\begin{aligned}
0<\varepsilon \lesssim & j^{p-1}\left(\varphi\left(p_{j}, q_{j}\right)^{\frac{m-1}{m}}\right)^{p-2} \varphi\left(p_{j}, q_{j}\right)^{\frac{2 m-4}{m}} \\
& +j^{p-1}\left(\varphi\left(p_{j}, q_{j}\right)^{\frac{m-1}{m}}\right)^{p-4}\left(\varphi\left(p_{j}, q_{j}\right)^{\frac{4 m-6}{m}}\right) \\
\sim & j^{p-1}\left(\varphi\left(p_{j}, q_{j}\right)^{\frac{1}{m}}\right)^{p(m-1)-2} .
\end{aligned}
$$

Since $m>p+2$, we have $(p(m-1)-2)\left(\frac{1}{m}\right)>p-1$. We arrive at a contradiction as $j \rightarrow \infty$.

The next lemma is proved in the Heisenberg group as Lemma 4.6 of [4]. The proof in Carnot groups is identical and omitted.

Lemma 5.9. Let $v$ be a $p$-harmonic function in $\Omega$. For each $\varepsilon \geq 0$, let $v_{\varepsilon}$ be the continuous $\varepsilon$-weak solution equal to $v$ on the boundary. Then $v_{\varepsilon} \rightarrow v$ pointwise as $\varepsilon \rightarrow 0$.

Combining the previous lemmas, we obtain the following consequence.

Lemma 5.10. Let $1<p<\infty$. 0-viscosity subsolutions are $p$-subharmonic. 0viscosity supersolutions are $p$-superharmonic and 0 -viscosity solutions are $p$-harmonic.

Proof. The last statement follows from the first two and the second follows from the first by replacing $u$ with $-u$. We let $u$ be a 0 -viscosity subsolution that is not $p$-subharmonic. Then there is a $p$-harmonic function $v$ so that $u \leq v$ on $\partial \Omega$ but for some $p \in \Omega$, we have $u(p)>v(p)$. For $\varepsilon \leq 1$, we let $v_{\varepsilon}$ be $\varepsilon$-weak solutions equal to $v$ on $\partial \Omega$ so that $u \leq v_{\varepsilon}$ on $\partial \Omega$. By Lemma 5.9 we conclude for some $\varepsilon$ near 0 , $u(p)>v_{\varepsilon}(p)$, contrary to Theorem 5.7 .

Combining Lemma 5.5 and 5.10, we have the following corollary. 
Corollary 5.11. Let $1<p<\infty$. Then 0-viscosity sub(super-)solutions to equation (5.3) and p-sub(super-)harmonic functions coincide. In particular, for $1<$ $p<\infty$, a function is p-harmonic if and only if it is a 0 -viscosity solution to equation (5.3).

We are then able to conclude the following comparison principle.

Theorem 5.12. Let $\varepsilon=0$. Let $v$ be a viscosity supersolution to equation (5.3) and let $u$ be a viscosity subsolution of equation (5.3) so that $u \leq v$ on $\partial \Omega$. Then $u \leq v$ in $\Omega$.

\section{References}

[1] Beatrous, F., T. Bieske, and J. Manfredi: The maximum principle for vector fields. The p-harmonic equation and recent advances in analysis. - Contemp. Math. 370, Amer. Math. Soc., Providence, RI, 2005, 1-9.

[2] Bellaïche, A.: The tangent space in sub-Riemannian geometry. - In: Sub-Riemannian geometry, edited by A. Bellaïche and J.-J. Risler, Progr. Math. 144, Birkhäuser, Basel, Switzerland, 1996, 1-78.

[3] Bieske, T.: On infinite harmonic functions on the Heisenberg group. - Comm. Partial Differential Equations 27:3-4, 2002, 727-762.

[4] Bieske, T.: Equivalence of weak and viscosity solutions to the $p$-Laplacian in the Heisenberg group. - Ann. Acad. Sci. Fenn. Math. 31, 2006, 363-379.

[5] Bourbaki, N.: Lie groups and Lie algebras. Chapters 1-3. - Elements of Mathematics (Berlin), Springer-Verlag, Berlin, 1989.

[6] Capogna, L., D. Danielli, and N. Garofalo: An embedding theorem and the Harnack inequality for nonlinear subelliptic equations. - Comm. Partial Differential Equations 18:9-10, 1993, 1765-1794.

[7] Crandall, M.: Viscosity solutions: a primer. - Lecture Notes in Math. 1660, Springer-Verlag, Berlin, 1997.

[8] Crandall, M., H. Ishit, and P.-L. Lions: User's guide to viscosity solutions of second order partial differential equations. - Bull. Amer. Math. Soc. 27:1, 1992, 1-67.

[9] Folland, G. B., and E. M. Stein: Hardy spaces on homogeneous groups. - Princeton Univ. Press, Princeton, NJ, 1982.

[10] Heinonen, J., and I. Holopainen: Quasiregular maps on Carnot groups. - J. Geom. Anal. $7: 1,1997,109-148$.

[11] Heinonen, J., T. Kilpeläinen, and O. Martio: Nonlinear potential theory of degenerate elliptic equations. - Oxford Math. Monogr., Oxford Univ. Press, New York, 1993.

[12] Jensen, R.: Uniqueness of Lipschitz extensions: minimizing the sup norm of the gradient. Arch. Rational Mech. Anal. 123, 1993, 51-74.

[13] Juutinen, P., P. Lindqvist, and J. Manfredi: On the equivalence of viscosity solutions and weak solutions for a quasi-linear equation. - SIAM J. Math. Anal. 33:3, 2001, 699-717.

[14] Kinnunen, J., and O. Martio: Nonlinear potential theory on metric spaces. - Illinois J. Math. 46:3, 2002, 857-883.

[15] WANG, C. Y.: The Aronsson equation for gradient minimizers of $L^{\infty}$-functionals associated with vector fields satisfying Hörmander's condition. - Trans. Amer. Math. Soc. 359, 2007, 91113. 